\title{
Antimicrobial photodynamic therapy minimizes the deleterious effect of nicotine in female rats with induced periodontitis
}

\author{
Erivan Clementino Gualberto Jr. ${ }^{1}$. Letícia Helena Theodoro ${ }^{1} \cdot$ Mariellén Longo $^{1}$ • \\ Vivian Cristina Noronha Novaes ${ }^{1} \cdot$ Maria José Hitomi Nagata ${ }^{1} \cdot$ Edilson Ervolino $^{2}$. \\ Valdir Gouveia Garcia ${ }^{1,3,4}$
}

Received: 8 April 2015 / Accepted: 13 October 2015 /Published online: 6 November 2015

(C) Springer-Verlag London 2015

\begin{abstract}
The aim of this study was to compare the use of antimicrobial photodynamic therapy (aPDT) as an adjunct to scaling and root planing (SRP) in the treatment of experimentally induced periodontitis in female rats that were systemically treated with or without nicotine. Female rats $(n=180)$ were divided into two groups: vehicle administration (Veh) and nicotine administration (Nic). Mini-pumps containing either vehicle or nicotine were implanted in the rats 30 days before the induction of experimental periodontitis (EP). EP was induced by placing a cotton ligature around the left mandibular first molar. After 7 days, the ligature was removed, and the rats were randomly divided into three treatment subgroups: SRP (only SRP), DL (SRP plus diode laser), and aPDT (SRP plus aPDT). The aPDT consisted of phenothiazine photosensitizer deposition followed by diode laser irradiation. Ten rats from each subgroup were euthanized at 7, 15, and 30 days after treatment. Alveolar bone loss (ABL) in the furcation region was evaluated using histological, histometric, and immunohistochemical analyses. The rats that were treated with nicotine showed more ABL compared to those treated with
\end{abstract}

Valdir Gouveia Garcia

vgouveia@foa.unesp.br

1 Group for the Research and Study of Laser in Dentistry, Department of Surgery and Integrated Clinic, Division of Periodontics, São Paulo State University (UNESP), Araçatuba, São Paulo, Brazil

2 Department of Basic Science, São Paulo State University (UNESP), Araçatuba, São Paulo, Brazil

3 Dental School of Barretos, University Center of the Educational Foundation of Barretos, Barretos, SP, Brazil

4 Faculdade de Odontologia de Araçatuba-UNESP, Rua José Bonifácio, 1193, 16015-050 Araçatuba, SP, Brazil vehicle. In both the Veh and Nic groups, SRP plus aPDT treatment resulted in reduced ABL, smaller numbers of both TRAP- and RANKL-positive cells, and higher numbers of PCNA-positive cells compared to SRP treatment alone. aPDT was an effective adjunctive therapy for the treatment of periodontitis in female rats regardless of whether they received nicotine.

Keywords Alveolar bone loss $\cdot$ Lasers $\cdot$ Nicotine . Periodontitis $\cdot$ Photochemotherapy $\cdot$ RANK ligand

\section{Introduction}

Tobacco smoking is strongly associated with both an increased risk of inflammatory disease, such as periodontitis, and increased disease severity [1]. Many of the undesirable effects of tobacco have been attributed to nicotine [2]. Differences in the rate of nicotine metabolism between men and women have been suggested, with women metabolizing nicotine more rapidly than men. This difference explains women's increased predisposition to addiction and difficulty in quitting smoking $[3,4]$. Among the reasons for this difference in metabolism is the influence of estrogen on CYP2A6 [5], the main enzyme responsible for the conversion of nicotine to its inactive metabolite, cotinine [6]. Additionally, in women, nicotine reduces estrogen levels and leads to the early onset of menopause [7,8].

Nicotine exerts potent effects on alveolar bone tissue neoformation and resorption, processes that substantially affect the progression of periodontal disease and the host response to treatment. In cells of the osteoblast lineage, nicotine suppresses proliferation and differentiation and inhibits the expression of key angiogenic and osteogenic mediators in a dose-dependent manner $[9,10]$. In contrast, in the cells of the 
osteoclast lineage, nicotine also stimulates the production of osteoclast-like cells in a dose-dependent manner [11], especially via upregulation of the macrophage colony-stimulating factor (M-CSF) [12]. Nicotine is also able to change another regulatory pathway related to osteoclastogenesis and osteoclastic activity, the RANK/RANKL/OPG system. RANKL, which is produced predominantly by cells of the osteoblast lineage, interacts with RANK expressed by osteoclast lineage cells, leading to increased osteoclastogenesis and osteoclastic activity $[13,14]$. OPG is a decoy receptor for RANKL and prevents its interaction with RANK [15]. Nicotine strongly influences this system by increasing the RANKL/OPG ratio $[16,17]$.

Mechanical removal of contaminants is usually the first mode of therapy recommended for periodontitis [18]. However, this therapy has limitations that may be due to several factors, such as tooth anatomy, tissue invasion by periodontal pathogens, and potential recolonization from other diseased sites [19]. Antimicrobial photodynamic therapy (aPDT) has recently emerged as an adjunct to scaling and root planing (SRP). aPDT can enhance the effectiveness of periodontal treatment through its bactericidal effects and the inactivation of bacterial virulence factors and host cytokines that impair periodontal restoration [20]. Studies in male rats that were systemically treated with nicotine [21] and in ovariectomized female rats [22] showed that aPDT was able to reduce bone loss and inflammatory immune responses in induced periodontitis. However, the effect of aPDT in intact female rats systemically treated with nicotine and with induced periodontitis requires further study.

Accordingly, the aim of this study was to assess the effect of aPDT, as an adjunct to SRP, on alveolar bone loss, inflammatory immune responses, the modulation and recruitment of osteoclasts, and periodontal repair in female rats with experimentally induced periodontitis under the effects of systemic nicotine treatment.

\section{Materials and methods}

This study was conducted with adult female Wistar rats. At the start of the study, the rats were 3 months of age and weighed $266 \pm 6.5 \mathrm{~g}$ (mean $\pm \mathrm{SD})$. After an acclimatization period, the estrous cycle was monitored, and the rats that exhibited abnormal cycles were removed. The remaining rats $(n=180)$ were randomly distributed into two groups: the Veh group $(n=90)$, which was administered vehicle $(0.9 \%$ sodium chloride), and the Nic group $(n=90)$, which was administered nicotine. All protocols were approved by the Institutional Review Board of Araçatuba Dental School, São Paulo State University, Araçatuba, São Paulo, Brazil (no. 2010/005074). For all surgical procedures, the rats were anesthetized with ketamine $\left(70 \mathrm{mg} / \mathrm{kg}\right.$; Vetaset ${ }^{\circledR}$, Fort Dodge Animal Health,
Fort Dodge, IA, USA) and xylazine (6 mg/kg; Rompum, Bayer do Brazil, São Paulo, SP, Brazil) via intramuscular injection.

\section{Estrous cycle and dosage of estrogen}

To confirm regularity, estrous cycles were monitored 2 weeks after the osmotic pump placement and 1 week after the periodontal treatment. Changes in vaginal smears during the 4 to 5 days of the estrous cycles were observed in all rats (data not shown) [22].

The estrogen level was measured by radioimmunoassay. Blood was collected from the rats by cardiac puncture at the time of euthanasia. Serum samples were analyzed with a CoatA-Count Estradiol kit (17 $\beta$-estradiol double antibody, KE2D; Siemens Healthcare Diagnostics Inc., Tarrytown, NY, USA) and measured for $60 \mathrm{~s}$ with a gamma counter (Packard Cobra TM II Auto Gamma Counter Packard Bioscience, Meriden, CT, USA). Three standard curves were analyzed, and all samples were compared to their closest standard curve. Standards and samples were analyzed in duplicate.

\section{Protocol for the administration of vehicle or nicotine}

Nicotine and vehicle were administered by osmotic minipumps. Thirty days before ligature placement, the rats were anesthetized, and osmotic mini-pumps (Alzet model \#2006, Durect Corp., Palo Alto, CA, USA) containing nicotine or vehicle were surgically inserted subcutaneously into the backs of the rats. The pumps delivered the solution at a rate of $0.17 \mu \mathrm{L} / \mathrm{h}$ for a period of up to 40 days. Nicotine tartrate (Sigma Chemical Co., St Louis, MO, USA) was dissolved in a sterile solution of $0.9 \%$ sodium chloride to deliver an average of $6 \mathrm{mg}$ nicotine $/ \mathrm{kg} /$ day. In both groups, the pumps were replaced after the first 40 days, and the new pumps remained until euthanasia [23].

\section{Experimental periodontitis protocol}

Thirty days after the mini-pumps were implanted, a cotton ligature in a submarginal position was applied to one left mandibular first molar of each rat in both groups to induce experimental periodontitis (EP). The ligatures were removed from all rats after 7 days. The rats in each group (Veh and Nic) were randomly assigned through a computer-generated table to one of three treatment subgroups: only SRP, SRP plus diode laser (DL), or SRP plus antimicrobial photodynamic therapy (aPDT). Each subgroup had 30 rats. All treatment procedures were performed by the same experienced operator (ECGJ). 


\section{SRP treatment}

SRP was performed with manual curettes (\#1-2 Mini Five Gracey curettes, Hu-Friedy, Chicago, IL., USA) using ten distal-mesial traction movements on both the buccal and lingual aspects. The furcation and interproximal regions of each tooth were scaled with the same curettes using cervicalocclusal traction movements [21,22].

\section{DL treatment}

The laser used in this study was an indium-galliumaluminum-phosphorous laser (TheraLase, DMC Equipment, São Carlos, SP, Brazil) with a wavelength of $660 \mathrm{~nm}$ and a spot size of $0.0283 \mathrm{~cm}^{2}$. Laser light was applied to the buccal and lingual aspects of the left mandibular first molar perpendicularly and in contact with the gingivae. The laser was activated at a power of $0.035 \mathrm{~W}$ for $12 \mathrm{~s}$ for each buccal and lingual aspect ( $24 \mathrm{~s}$ per tooth), with $0.14 \mathrm{~J}$ per point of energy and an energy density of $14.82 \mathrm{~J} / \mathrm{cm}^{2}$. Each tooth received a total energy density of $29.64 \mathrm{~J} / \mathrm{cm}^{2}$ [22].

\section{aPDT treatment}

For the aPDT treatment, SRP of the left mandibular first molar was performed. Then, $1 \mathrm{~mL}$ of a 7-amino-8-methylphenothiazin-3-ylidene-dimethyl-ammonium (phenothiazine; Sigma Chemical Co., St. Louis, MO, USA) solution $(100 \mu \mathrm{g} /$ $\mathrm{mL}$ ) was applied, followed by DL treatment. The phenothiazine solution $(1 \mathrm{~mL})$ was slowly dropped into the periodontal pocket around the left mandibular first molar using a syringe and an unbeveled needle $(13 \times 0.45 \mathrm{~mm})$. After $1 \mathrm{~min}$ of phenothiazine application, the laser was applied to each buccal and lingual aspect of the left mandibular first molar perpendicularly and in contact with the gingivae. The laser parameters were the same as those described above [22].

\section{Experimental periods}

Ten rats from each treatment subgroup were euthanized 7, 15, and 30 days after the periodontal treatment via a lethal dose of thiopental (150 mg/kg; Cristália, Itapira, SP, Brazil). Their jaws were removed and fixed with $4 \%$ formaldehyde in $0.1 \mathrm{M}$ phosphate buffer ( $\mathrm{pH} 7.4$ ) for $48 \mathrm{~h}$.

\section{Laboratory procedures}

The specimens were demineralized in a solution of $10 \%$ EDTA (Sigma Chemical Co., St Louis, MO, USA), subjected to conventional histological processing, and embedded in paraffin. Semi-serial sections $(4 \mu \mathrm{m})$ were obtained in the latero-lateral direction so that the mandibular first molars were sectioned along the longitudinal axis. Some sections were stained with hematoxylin and eosin (HE) or Masson Trichrome (MT). Other sections were subjected to the indirect immunoperoxidase method with the following primary antibodies: anti-PCNA (VP 980-Vector Laboratories, Burlingame, CA, USA), anti-TRAP (goat anti-TRAPSC 30833, Santa Cruz Biotechnology, Santa Cruz, CA, USA), anti-RANKL (goat anti-RANKL-SC 7628, Santa Cruz Biotechnology, Santa Cruz, CA, USA), and anti-OPG (goat anti-OPG-SC 8468, Santa Cruz Biotechnology, Santa Cruz, CA, USA). The immunohistochemical procedure followed the protocol described by Garcia et al. [24].

\section{Histopathological analysis}

The sections stained with HE were analyzed by a certified histologist blinded to the treatments (EE). The following parameters were examined: nature and extension of the inflammatory process; presence of necrotic tissue; presence, extent, and nature of resorption of the bone, cementum, and dentin; state of the vasculature; structure of the extracellular matrix of the periodontal tissues; and cellular pattern of the periodontal tissues. The entire furcation region of the left mandibular first molar was analyzed.

\section{Histometric analysis}

The sections stained with TM were used to assess alveolar bone loss (ABL) in the furcation region. One trained examiner (ML), who was blinded to the groups and treatments, selected the sections for histometric analysis. Five equidistant sections were selected from each specimen and imaged using a digital camera coupled to a light microscope (AxioStar Plus, Carl Zeiss MicroImaging Gmb, 37030 Gottingen, Germany). Another calibrated examiner (ECGJ), who was blinded to the groups and treatments, conducted the histometric analysis. At $\times 50$ magnification, an image analysis system (AxioVision 4.8.2, Carl Zeiss MicroImaging GmbH, 07740 Jena, Germany) was used to measure the distance between the outer surface of the cementum and the boundary of the alveolar bone crest to determine the area of $\mathrm{ABL}\left(\mathrm{mm}^{2}\right)[21,22,24]$. ABL was measured three times by the same examiner on different days to reduce variations in the data. The mean values were averaged and compared statistically.

\section{Immunohistochemical analysis}

An examiner who was blinded to the groups and treatments (EE) conducted the immunohistochemical analyses. The 
Fig. 1 Photomicrographs showing the histological appearance of the furcation region of the left mandibular first molar at 7 days in the Veh (a-f) and Nic (g-l) groups after treatment with SRP $(\mathbf{a}, \mathbf{d}, \mathbf{g}$, and $\mathbf{j})$, SRP plus DL (b, e, h, and $\mathbf{k}$ ), and SRP plus aPDT (c, f, i, and $\mathbf{l})$

Abbreviations and symbols: $a b$, alveolar bone; asterisks, inflammatory infiltrate. Staining: hematoxylin and eosin (HE). Original magnification: $\mathbf{a}-\mathbf{c}$ and $\mathbf{g}-\mathbf{i}, \times 100 ; \mathbf{d}-\mathbf{f}$ and $\mathbf{j}-\mathbf{l}, \times 250$. Scale bars: a-c and $\mathbf{g}-\mathbf{i}, 250 \mu \mathrm{m}$; $\mathbf{d}-\mathbf{f}$ and $\mathbf{j}-\mathbf{l}, 100 \mu \mathrm{m}$
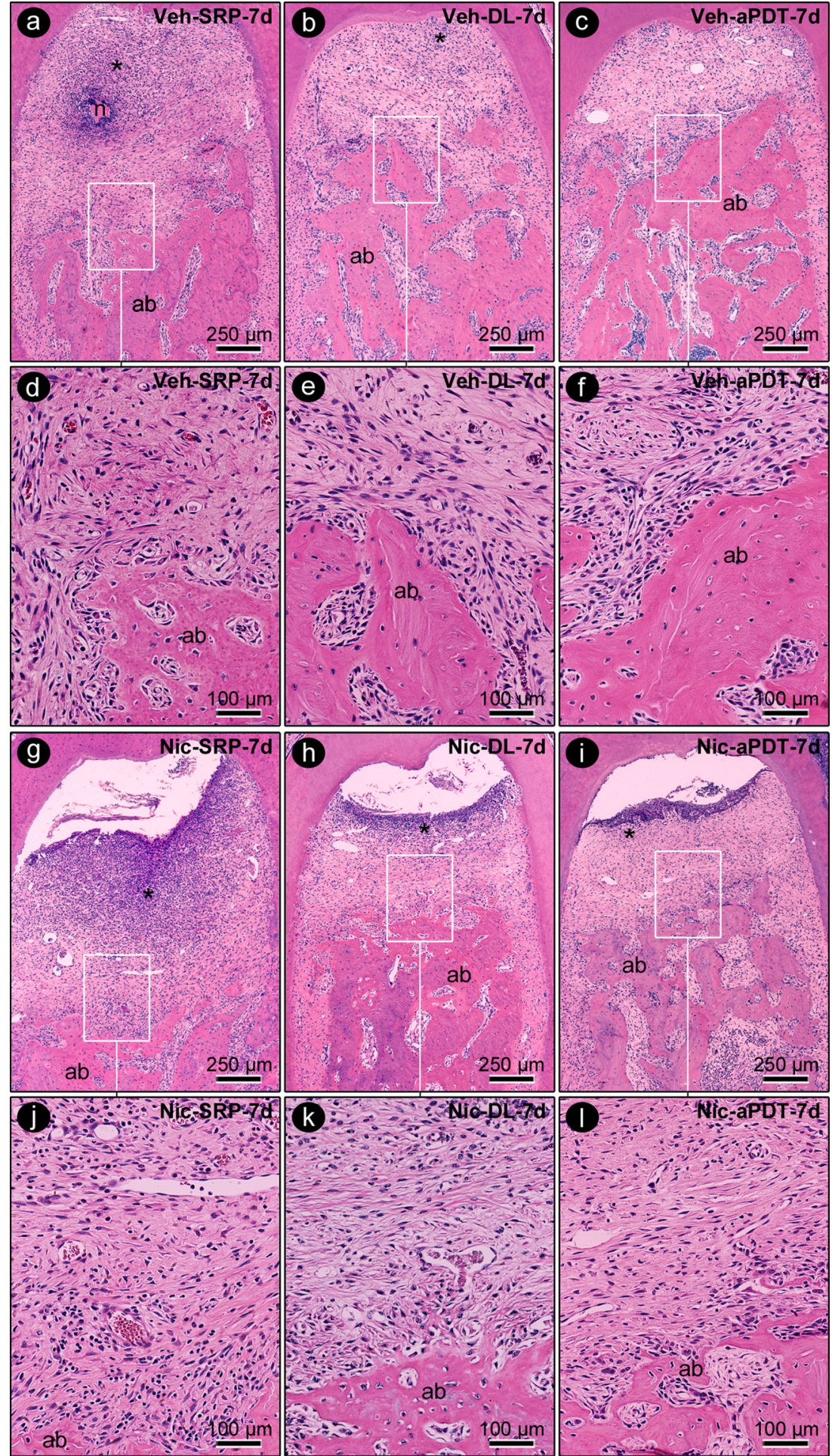

values for each section were measured three times by the same examiner on different days to reduce variations in the data [21, $22,24]$.

Immunolabeling for RANKL and OPG was analyzed in the entire furcation region of the mandibular first molar under $\times 400$ magnification. A semi-quantitative analysis was performed. Three histological sections from each animal were used, and the immunolabeling criteria followed those of Garcia et al. [24] as follows: score 0-no marking $(0 \%)$, score 1 -weak marking $(<25 \%$ of cells $)$, score 2 -moderate marking $(<50 \%$ of cells $)$, and score 3 strong intensity ( $<75 \%$ of cells). 
Fig. 2 Photomicrographs showing the histological appearance of the furcation region of the left mandibular first molar at 15 days in the Veh $(\mathbf{a}-\mathbf{f})$ and Nic (g-l) groups after treatment with $\operatorname{SRP}(\mathbf{a}, \mathbf{d}, \mathbf{g}$, and $\mathbf{j})$, SRP plus DL (b, e, h, and $\mathbf{k})$, and SRP plus aPDT (c, $\mathbf{f}, \mathbf{i}$, and $\mathbf{l})$.

Abbreviations and symbols: $a b$, alveolar bone; asterisks, inflammatory infiltrate. Staining: hematoxylin and eosin (HE). Original magnification: $\mathbf{a}-\mathbf{c}$ and $\mathbf{g}-\mathbf{i}, \times 100 ; \mathbf{d}-\mathbf{f}$ and $\mathbf{j}-\mathbf{l}, \times 250$. Scale bars: $\mathbf{a}-\mathbf{c}$ and $\mathbf{g}-\mathbf{i}, 250 \mu \mathrm{m}$; $\mathbf{d}-\mathbf{f}$ and $\mathbf{j}-\mathbf{l}, 100 \mu \mathrm{m}$
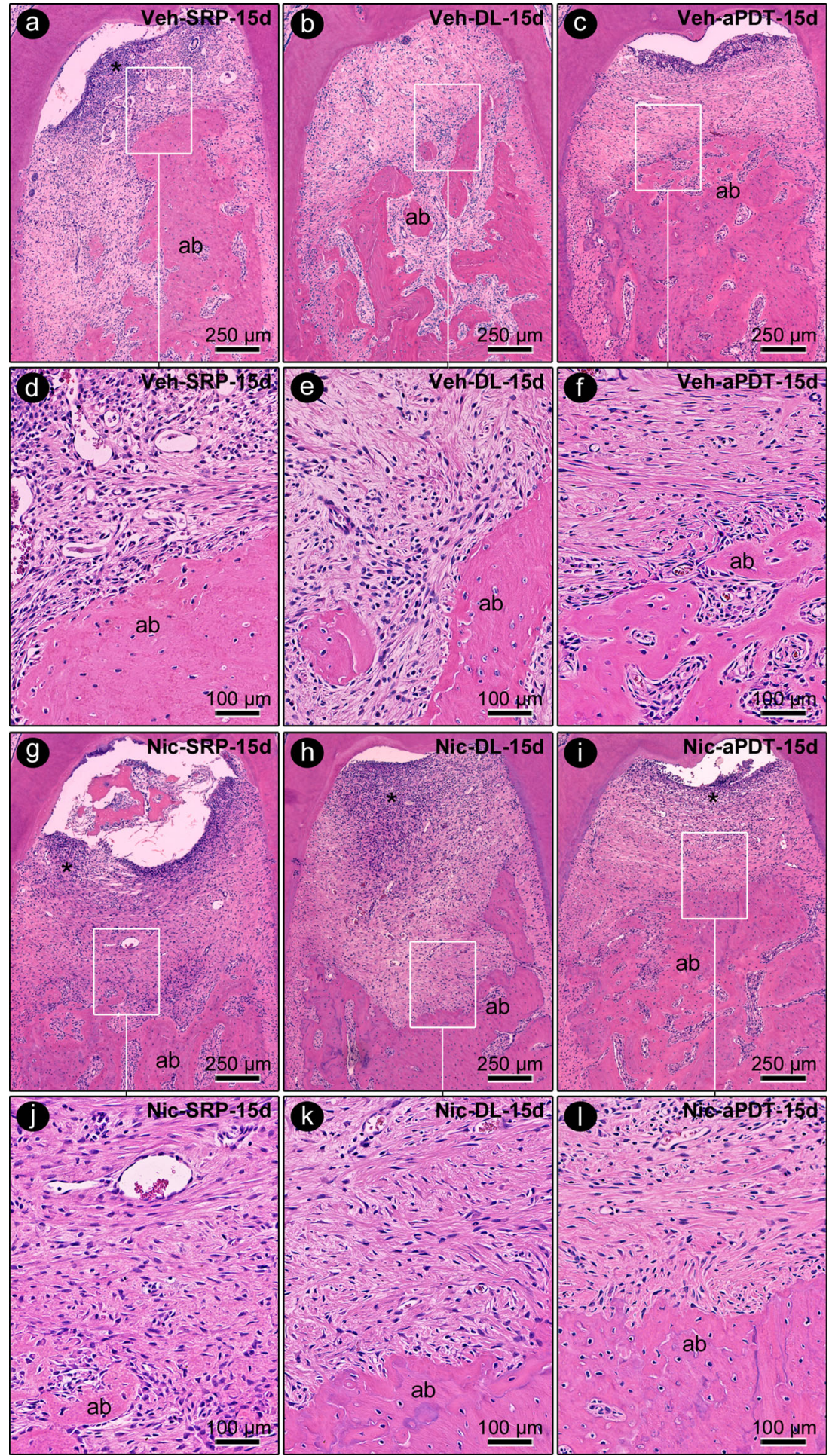

PCNA- and TRAP-positive cells were analyzed within a $1000 \times 1000 \mu \mathrm{m}$ portion of the central area of the interradicular septum under $\times 200$ magnification. The coronary limit was the bone crest, which was spanned apically for a distance of $1000 \mu \mathrm{m}$. Quantitative analysis for TRAP and PCNA was performed in five sections from each animal. Only the TRAPpositive, multinucleated cells were analyzed [24].

\section{Intra-examiner reproducibility}

Before the histometric and immunohistochemical analyses were performed, the examiner was trained and then he calibrated the analyses by performing double measurements of 30 specimens with a 1-week interval. Pearson's correlation coefficient revealed a very high correlation $(0.95)$ between the two 
Fig. 3 Photomicrographs showing the histological appearance of the furcation region of the left mandibular first molar at 30 days in the Veh $(\mathbf{a}-\mathbf{f})$ and the Nic (g-l) groups after treatment with SRP (a, d, $\mathbf{g}$, and $\mathbf{j})$, SRP plus DL (b, e, h, and $\mathbf{k})$, and SRP plus aPDT (c, $\mathbf{f}, \mathbf{i}$, and $\mathbf{l})$.

Abbreviations and symbols: $a b$, alveolar bone; asterisks, inflammatory infiltrate. Staining: hematoxylin and eosin (HE). Original magnification: $\mathbf{a}-\mathbf{c}$ and $\mathbf{g}-\mathbf{i}, \times 100 ; \mathbf{d}-\mathbf{f}$ and $\mathbf{j}-\mathbf{l}, \times 250$. Scale bars: $\mathbf{a}-\mathbf{c}$ and $\mathbf{g}-\mathbf{i}, 250 \mu \mathrm{m}$; $\mathbf{d}-\mathbf{f}$ and $\mathbf{j}-\mathbf{l}, 100 \mu \mathrm{m}$
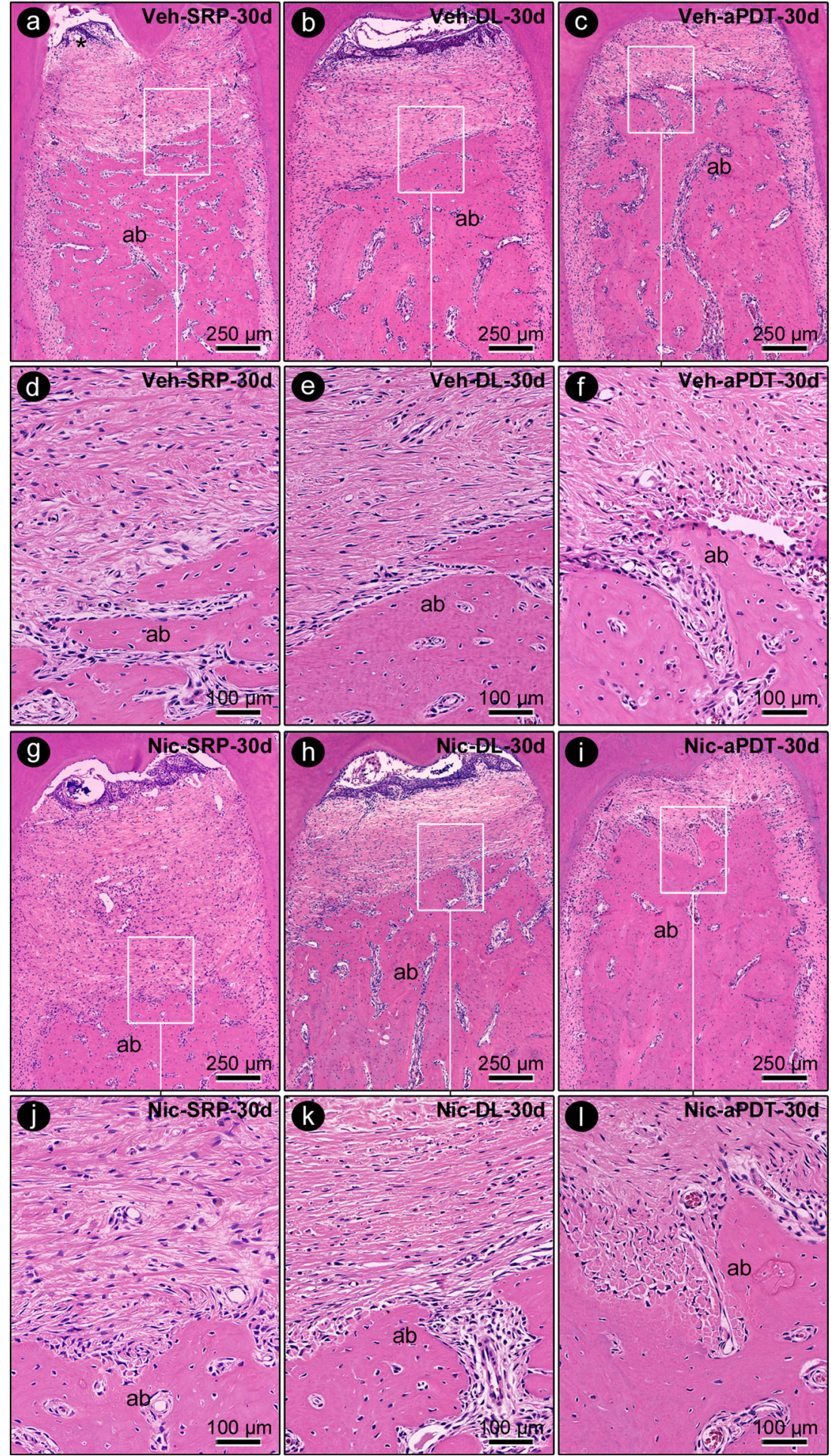

sets of measurements for both the histometric and immunohistochemical analyses.

\section{Statistical analysis}

The results demonstrated that with a sample size of 10 $(p<0.05)$, the power of the study would be $95 \%$. The hypothesis that neither ABL nor the number of TRAP- and PCNA-positive cells in the furcation region would differ between the groups and time points was tested using a statistical software (Bioestat 5.3, Manaus, AM, Brazil). The normality of the histometric data was analyzed using the Shapiro-Wilk test. The intra- and inter-group analyses were performed with analysis of variance (ANOVA) $(p<0.05)$. When ANOVA 
detected a significant difference, multiple comparisons were performed using Tukey's test $(p<0.05)$ for ABL- and TRAPpositive cells. Bonferroni's test $(p<0.05)$ was used for PCNApositive cells.

\section{Results}

\section{Hormone radioimmunoassay}

At the time of euthanasia, the mean serum concentration of $17 \beta$-estradiol did not statistically differ between the Veh $(45.74 \mathrm{pg} / \mathrm{mL})$ and $\mathrm{Nic}(43.08 \mathrm{pg} / \mathrm{mL})$ groups.

\section{Histological analyses}

SRP subgroup At 7 and 15 days, the Veh and Nic groups that were treated with SRP exhibited connective tissue with intense inflammatory infiltrate, which was composed mainly of neutrophils, in the furcation regions (Figs. 1a, d, g, j and $2 a, d, g, j)$. After 30 days, the volume of inflammatory infiltrate was reduced in the Veh group, and the connective tissue and bone showed signs of repair (Fig. 3a, d). At the same point in time, the Nic group showed a pattern of tissue disruption similar to the one observed at 15 days (Fig. $3 g$, j). At all time points, the magnitude of the inflammatory response, tissue disorganization, and ABL level were greater in the Nic group than in the Veh group (Figs. 1g, j; 2g, j; and 3g, j).

DL subgroup At 7 days, the Veh group showed a moderate inflammatory infiltrate in the furcation region (Fig. 1b, e). At 15 and 30 days, the inflammatory infiltrate was greatly decreased, the connective tissue showed a moderate amount of fibroblasts and collagen fibers, and the bone tissue showed fewer signs of active resorption (Figs. 2b, e and 3b, e). The Nic group, compared with the Veh group, showed more intense inflammation and tissue destruction after 7 days (Fig. 1h, k). At 15 and 30 days, signs of tissue repair were tangible; however, the delay in repair was clear (Figs. $2 \mathrm{~h}, \mathrm{k}$ and $3 \mathrm{~h}, \mathrm{k})$.

aPDT subgroup At 7 days, both the Veh and Nic groups showed mild infiltration into the connective tissue of the furcation region; this infiltrate was rich in fibroblasts and exhibited a moderate amount of collagen fibers (Fig. 1c, f, i, l). At 15 days, tissue repair signals and a decrease in inflammation were clear in both groups; however, the tissues were more mature in the Veh group (Fig. 2c, f, i, 1). At day 30, the histological results of both the Veh and Nic groups were very similar: the inflammatory infiltrate was very mild in the fibrous connective tissue, with moderate quantities of fibroblasts and blood vessels. The bone tissue showed few areas of active resorption and many areas of bone formation (Fig. 3c, f, i, 1).

\section{Histometric assessment}

Inter-group assessment demonstrated that, at 7 and 15 days, the Veh group exhibited reduced ABL compared to the Nic group $(p<0.05)$. Intra-group assessment revealed that at 7 and 15 days, the rats in the Veh group that were treated with DL and aPDT exhibited decreased ABL compared with those treated with SRP $(p<0.01)$. In the Nic group, there was less $\mathrm{ABL}$ at $7,15(p<0.01)$, and 30 days $(p<0.05)$ in the rats that were treated with aPDT compared with those treated with SRP. At 15 and 30 days $(p<0.05)$, there was less ABL in the rats in the Nic group that were treated with DL compared to those treated with SRP (Fig. 4).

\section{Immunohistochemical assessment}

The immunohistochemical method used to detect PCNA, TRAP, RANKL, and OPG showed high specificity, which was confirmed by the total absence of labeling in the negative control. The immunoreactive cells were brownish in color. Positive immunoreactivity was
Fig. 4 Graphic showing the mean \pm SD of alveolar bone loss $\left(\mathrm{mm}^{2}\right)$ in the furcation region of the left mandibular first molar according to group, treatment, and time point. Symbols: asterisks, significantly different from SRP treatment, for the same group and period (ANOVA and Tukey, $p<0.05$ ); daggers, significantly different from the Veh group, for the same treatment and period (ANOVA and Tukey, $p<0.05$ )

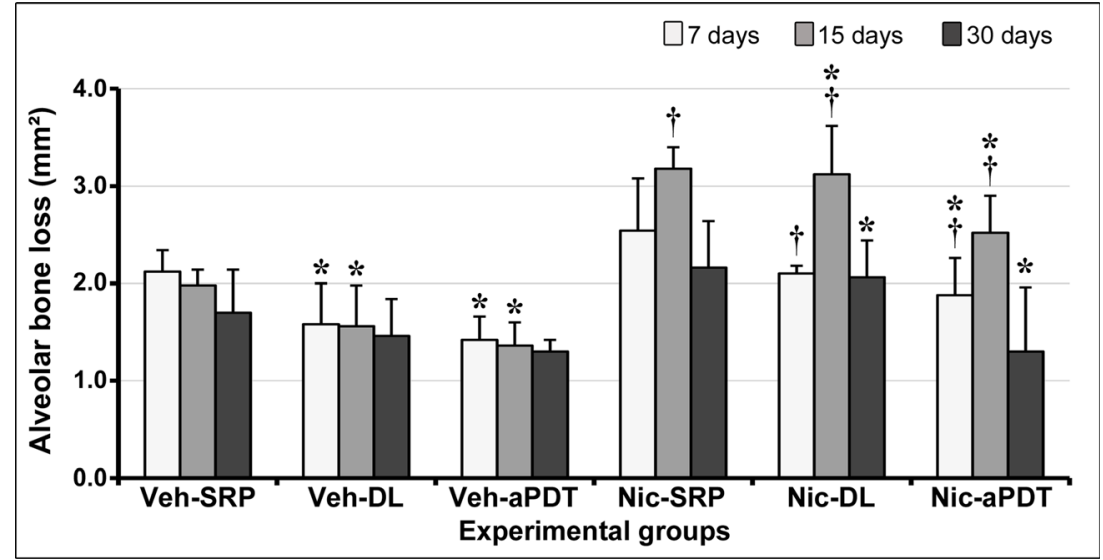



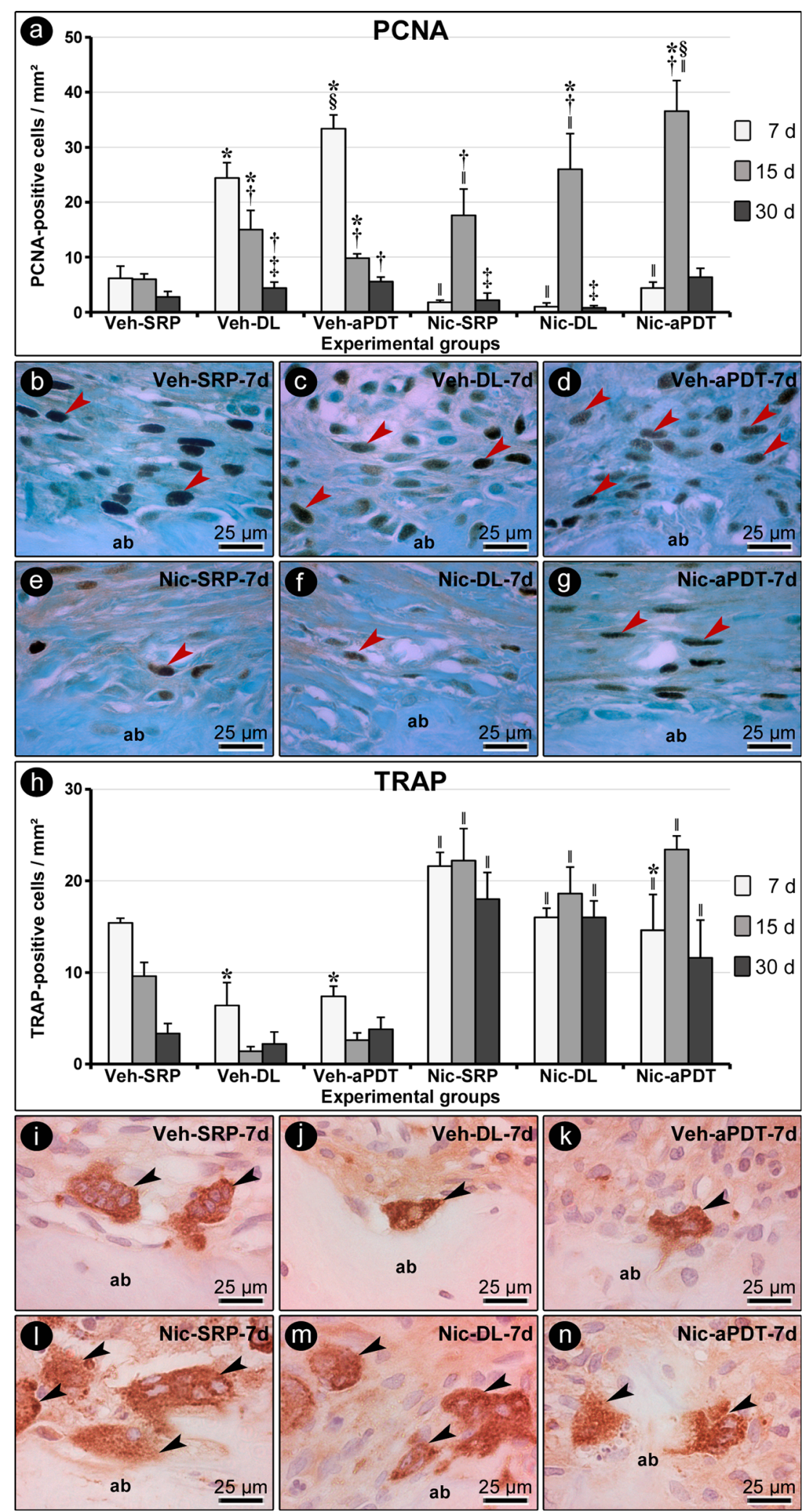

predominantly found in cells located in the connective tissue and bone for PCNA, in osteoclasts for TRAP, and in osteoblasts for RANKL and OPG.
PCNA The Veh group showed a higher number of PCNApositive cells at 7 days compared to the Nic group $(p<0.05)$. At 15 days, the Nic group showed a higher number of PCNA- 
4 Fig. 5 Immunolabeling for PCNA and TRAP in the furcation region of the left mandibular first molar in different experimental groups. a Graphic showing the mean $\pm \mathrm{SD}$ of the number of PCNA-positive cells according to group, treatment, and time point. b-g Photomicrographs showing PCNA-positive cells (black arrows) in the Veh (b-d) and Nic (e-g) groups after treatment with SRP (b and e), SRP plus DL (c and f), and SRP plus aPDT (d and $\mathbf{g}$ ) at 7 days. $\mathbf{h}$ Graphic showing the mean \pm SD of the number of TRAP-positive cells according to group, treatment, and time point. $\mathbf{i}-\mathbf{n}$ Photomicrographs showing TRAP-positive cells (white arrows) in the Veh (i-k) and Nic (1-n) groups after treatment with SRP (i and $\mathbf{l}$ ), SRP plus DL ( $\mathbf{j}$ and $\mathbf{m}$ ), and SRP plus aPDT ( $\mathbf{k}$ and $\mathbf{n}$ ) at 7 days. Abbreviations and symbols: $a b$, alveolar bone; black arrows, TRAPpositive osteoclasts; red arrows, PCNA-positive cells; asterisks, significantly different from SRP treatment for the same group and time point; daggers, significantly different from day 7 for the same group and treatment; double daggers, significantly different from day 15 for the same group and treatment; section sign, significantly different from DL treatment for the same group and time point; double vertical lines, significantly different from the Veh group for the same treatment and time point (PCNA: ANOVA and Bonferroni; TRAP: ANOVA and Tukey, $p<0.05)$. Counterstaining: Fast green $(\mathbf{b}-\mathbf{g})$ or Harris hematoxylin (i-n). Original magnification: $\mathbf{b}-\mathbf{g}$ and $\mathbf{i}-\mathbf{n}, \times 1000$; scale bars: $\mathbf{b}-\mathbf{g}$ and $\mathbf{i}-\mathbf{n}, 25 \mu \mathrm{m}$

positive cells compared to the Veh group $(p<0.05)$. In the Veh group, the rats that were treated with aPDT showed a greater number of PCNA-positive cells on day 7 compared to those treated with SRP $(p<0.05)$. At 15 days, the rats of both experimental groups that were treated with aPDT showed a higher number of PCNA-positive cells than the rats treated with SRP $(p<0.05)$. In the Veh group, the rats that were treated with DL showed a higher number of PCNA-positive cells compared with those treated with SRP at 7 and 15 days $(p<0.05)$. At 15 days, the rats in the Nic group also showed a higher number of PCNA-positive cells than those treated with SRP $(p<0.05)$ (Fig. 5a, b, c, d, e, f, g).

TRAP Compared with the Veh group, the Nic group showed a greater number of TRAP-positive cells at all post-treatment time points $(p<0.05)$. In both the Veh and Nic groups, the rats that were treated with SRP plus aPDT showed a lower number of TRAP-positive cells at 7 days compared to those treated with SRP $(p<0.05)$. In the Veh group, the rats that were treated with DL showed a lower number of TRAP-positive cells at 7 days compared to those treated with SRP $(p<0.05)$ (Fig. 5h, $\mathrm{i}, \mathrm{j}, \mathrm{k}, \mathrm{l}, \mathrm{m}, \mathrm{n})$.

RANKL In the Veh group, moderate immunostaining (score 2) was most prevalent, except at 15 and 30 days after SRP plus aPDT treatment, when low immunostaining (score 1) was prevalent (Fig. 6a, b, c). In the Nic group, high immunostaining (score 3) was most prevalent after SRP treatment, and moderate immunostaining (score 2) was observed after SRP plus aPDT. In the subgroup that was treated with SRP plus DL, the immunostaining was predominantly high (score 3 ) at 7 days and moderate (score 2) at 15 and 30 days (Fig. 6d, e, f).
OPG In the Veh and Nic groups, low immunostaining (score 1) was most prevalent, except at 30 days in the SRP plus DLtreated rats of the Veh group and in the rats of both the Veh and Nic groups that were treated with SRP plus aPDT (Fig. 6g, h, $\mathrm{i}, \mathrm{j}, \mathrm{k}, \mathrm{l})$.

\section{Discussion}

In this study, nicotine accelerated ABL by increasing and maintaining the number of osteoclasts. On the other hand, when combined with SRP, aPDT minimized the deleterious effects of nicotine, especially on bone metabolism, by regulating the RANK/RANKL/OPG system. The radioimmunoassay showed that estrogen concentrations were within the physiological range [25]. Although the effect of nicotine on estrogen was not a focus of this study, nicotine did not negatively impact estrogen levels, which conflicts with the results of Raval et al. [26].

More ABL was observed in the rats that were treated with nicotine than in the rats that were treated with vehicle, which corroborates the results of previous studies [21, 27]. This effect was greater at 7 and 15 days. Nicotine inhibits apoptosis in certain cell types, such as osteoclasts; as a result, these cells live longer and are able to continue resorption long after the end of their normal life cycle [11]. Furthermore, nicotine significantly reduces trabecular bone volume and the trabecular thickness of the mineralized surface, the mineral apposition rate, and new bone formation, leading to an increase in osteoclast area [28]. Nicotine negatively affected the tissue response to the periodontal treatments used in this study.

The female rats that were treated with aPDT showed reduced ABL compared with the rats that were treated with SRP alone; this finding was observed in the Veh group at 7 and 15 days and in the Nic group at all time points. However, the ABL effect was statistically significant only in the Veh group at 7 days. The beneficial effects of the use of aPDT as an adjunct to mechanical treatments for periodontal disease are most likely due to the activity of aPDT against periodontopathogenic bacteria [29]. During the process of aPDT, the photosensitizer (PS) absorbs energy directly from the laser and transfers it to oxygen molecules, resulting in the formation of reactive oxygen species (type I reaction) and singlet oxygen (type II reaction) [30, 31]. Gram-positive bacteria are more susceptible to aPDT, but with the selection of the appropriate PS and wavelength, gram-negative bacteria can also become susceptible [32]. Phenothiazines (which are used as PSs) are cationic molecules composed of a tricyclic aromatic ring. These structural characteristics enable PSs to absorb light at a wavelength of 600-660 $\mathrm{nm}$ [33]. The photosensitizer toluidine blue O (PS TBO) has been successfully used in aPDT against various types of bacteria [34, 35]. Although it is toxic to bacteria, at a concentration of $100 \mu \mathrm{g} /$ 
$\mathrm{mL}$, TBO is safe to periodontal tissues. Studies by our research group have shown that TBO at this concentration reduced the inflammatory infiltrate in the periodontal tissues and lowered the inflammatory immune response [24].

In this study, nicotine affected the RANKL/RANK/OPG axis, upregulated RANKL, and downregulated OPG. The RANKL/RANK/OPG axis plays an important role in osteoclast differentiation and bone remodeling $[15,36]$. An increase in RANKL leads to the activation of RANK, which in turn leads to increased osteoclastogenesis, the activation of mature osteoclasts and the inhibition of apoptosis [11, 15, 36, 37]. These effects could explain the larger increase of TRAP-positive cells that was observed in the Nic group. Any deregulation of this axis can alter bone metabolism and result in either the loss or gain of bone mass $[11,36]$. In a healthy periodontium, RANKL is upregulated, whereas OPG is downregulated in periodontitis; this difference results in a higher RANKL/OPG ratio [37]. The fact that nicotine can modulate osteoclast stimulation may partially explain the rapid increase of bone loss in the periodontium [11].
In the present study, aPDT affected the RANK/RANKL/ OPG system, resulting in RANKL downregulation, OPG upregulation, and, consequently, lower numbers of TRAPpositive cells. This effect shows that aPDT reversed the nicotine-induced changes in this system, making it compatible with periodontal health. The histological analysis showed that aPDT decreased the inflammatory infiltrate, which may explain the downregulation of RANKL and upregulation of OPG. These results corroborate those of other studies [21, 22, 24].

The initial response to bacterial infection involves a local inflammatory reaction that activates the innate immune system [37, 38]. During an inflammatory response, cytokines, chemokines, and other mediators can induce osteoclastogenesis by increasing the expression of RANKL and decreasing the expression of OPG in osteoblasts/stromal cells [39, 40]; aPDT can impact the gingival inflammation during chronic periodontitis and lead to a specific decrease in antigenpresenting cells [41]. Most likely, the antimicrobial effect of aPDT inhibits the exacerbated inflammatory response to local
Fig. 6 Immunostaining for RANKL and OPG in the furcation region of the left mandibular first molar in the different experimental groups. a-f Photomicrographs showing RANKL-positive cells (arrows) in the Veh $(\mathbf{a}-\mathbf{c})$ and Nic $(\mathbf{d}-\mathbf{f})$ groups after treatment with SRP (a, d), SRP plus DL (b, e), and SRP plus aPDT $(\mathbf{c}, \mathbf{f})$ at 7 days. $\mathbf{g}$ I Photomicrographs showing OPG-positive cells (arrows) in the Veh $(\mathbf{g}-\mathbf{i})$ and $\mathrm{Nic}(\mathbf{j}-\mathbf{l})$ groups after treatment with SRP $(\mathbf{g}, \mathbf{j})$, SRP plus DL (h, $\mathbf{k})$, and SRP plus $\operatorname{aPDT}(\mathbf{i}, \mathbf{l})$ at 30 days.

Abbreviations and symbols: $a b$, alveolar bone; black arrows, immunolabeled cells. Counterstaining: Harris hematoxylin. Original magnification $\times 1000$; scale bars $25 \mu \mathrm{m}$
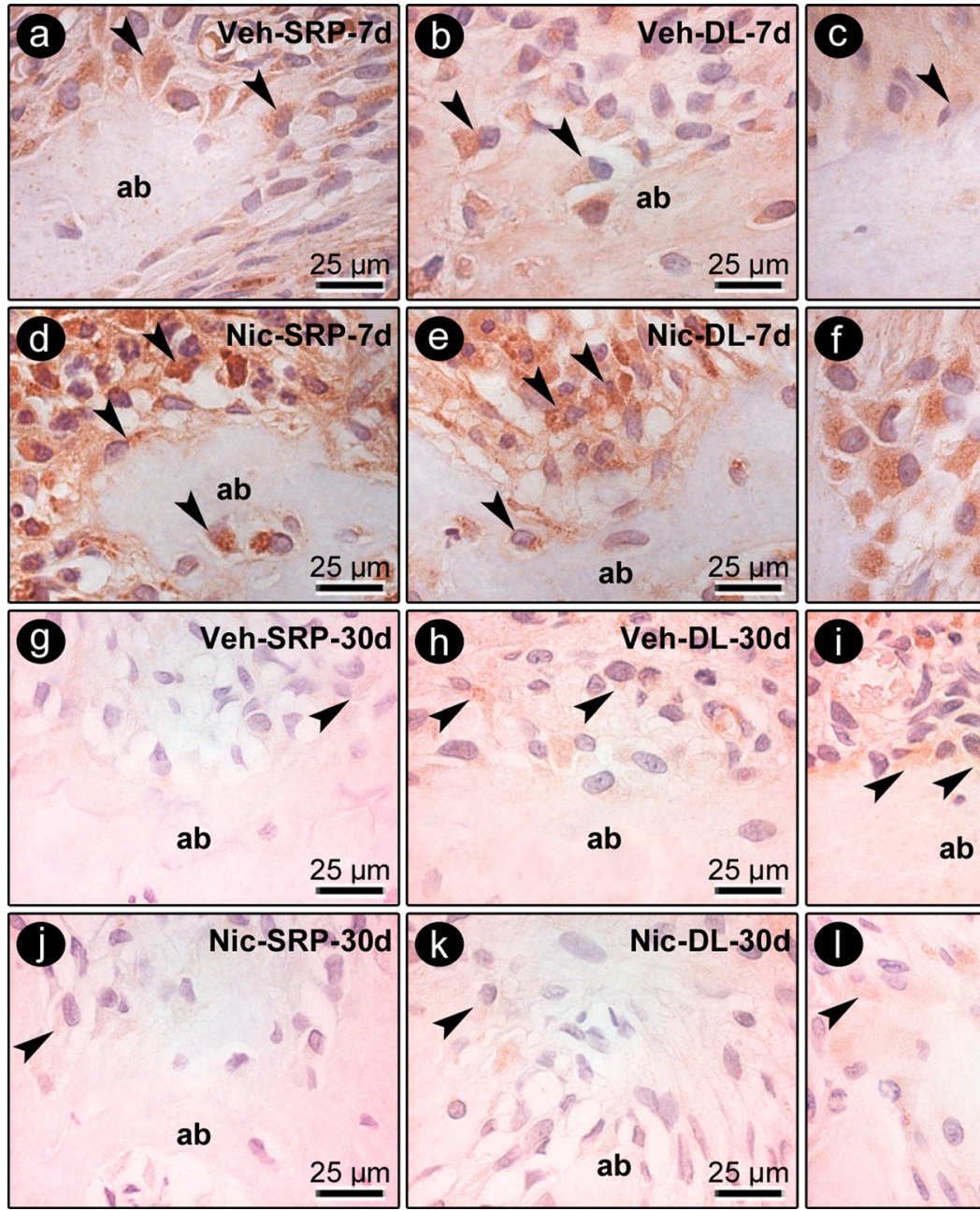

Veh-aPDT-7d
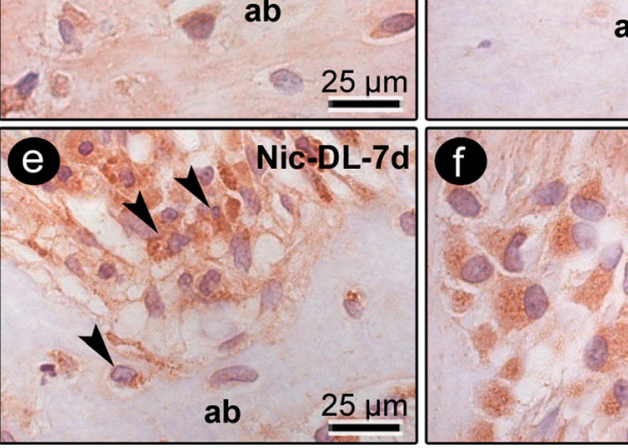

ab
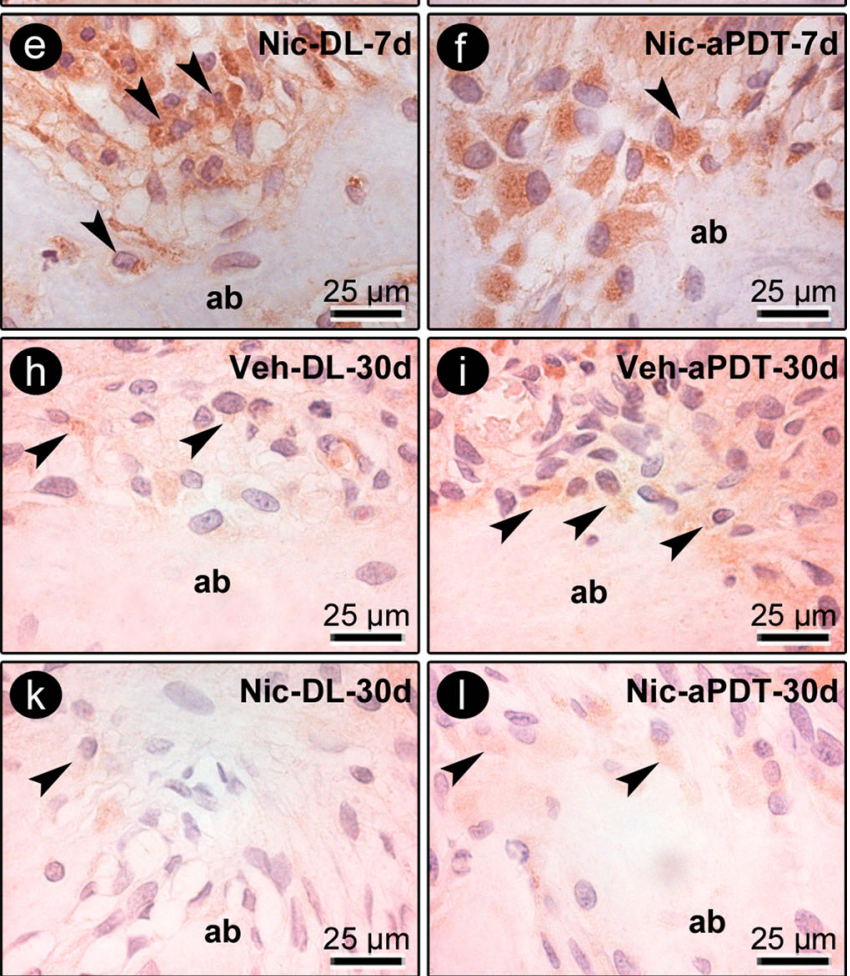
bacterial infections and, consequently, the cascade of events that leads to osteoclastogenesis and bone loss via the RANK/RANKL/OPG axis.

In this study, nicotine delayed the peak cell proliferation that is essential for repair. The results showed that there were more PCNA-positive cells at 7 days in the Veh group and at 15 days in the Nic group. However, the rats in both the Veh and Nic groups that were treated with DL and aPDT showed a high number of PCNA-positive cells. By leading to downregulation of RANKL and, hence, to a small number of TRAPpositive cells, aPDT created a more favorable environment for repair. These results are in general agreement with those of Garcia et al. [22], who showed that the number of PCNApositive cells in the periodontal ligament was significantly higher in rats treated with aPDT compared to SRP alone.

\section{Conclusion}

In this study, aPDT was an effective adjunct to SRP treatment, as demonstrated by the modulation of the RANK/RANKL/ OPG system, the decrease in the number of osteoclasts, and the high numbers of PCNA-positive cells in the furcation region that were observed in the female rats that were treated with or without nicotine. Thus, aPDT could be considered an effective adjuvant and indicated for the treatment of periodontitis in women under the systemic effects of nicotine; however, additional experimental studies in animals and clinical trials in women must be performed.

Acknowledgments This study was financially supported by the São Paulo Research Foundation (FAPESP), São Paulo, SP, Brazil (FAPESP processes no. 2011/00936-0 and no. 2010/15094-2). The authors are grateful to Prof. Dr. Guilherme de Paula Nogueira (Faculty of Veterinary Medicine, UNESP, Araçatuba, SP, Brazil) for his guidance in the use of radioimmunoassay. The authors report no conflicts of interest related to this study.

Compliance with Ethical Standards All protocols were approved by the Institutional Review Board of Araçatuba Dental School, São Paulo State University, Araçatuba, São Paulo, Brazil (no. 2010/005074).

\section{References}

1. Johannsen A, Susin C, Gustafsson A (2014) Smoking and inflammation: evidence for a synergistic role in chronic disease. Periodontol 64:111-126

2. Hebert R (2003) What's new in nicotine \& tobacco research? Nicotine Tob Res 5:427-433

3. Benowitz NL, Lessov-Schlaggar CN, Swan GE, Jacob P (2006) 3 rd. Female sex and oral contraceptive use accelerate nicotine metabolism. Clin Pharmacol Ther 79:480-488

4. Johnstone E, Benowitz N, Cargill A et al (2006) Determinants of the rate of nicotine metabolism and effects on smoking behavior. Clin Pharmacol Ther 80:319-330
5. Higashi E, Fukami T, Itoh $\mathrm{M}$ et al (2007) Human CYP2A6 is induced by estrogen via estrogen receptor. Drug Metab Dispos 35:1935-1941

6. Nakajima M, Yamamoto T, Nunoya K et al (1996) Role of human cytochrome P4502A6 in C-oxidation of nicotine. Drug Metab Dispos 24:1212-1217

7. Jensen J, Christiansen C, Rødbro P (1985) Cigarette smoking, serum estrogens, and bone loss during hormone-replacement therapy early after menopause. N Engl J Med 17(313):973-975

8. Mueck AO, Seeger H (2005) Smoking, estradiol metabolism and hormone replacement therapy. Curr Med Chem Cardiovasc Hematol Agents 3:45-54

9. Ma L, Zwahlen RA, Zheng LW, Sham MH (2011) Influence of nicotine on the biological activity of rabbit osteoblasts. Clin Oral Implants Res 22:338-342

10. Kim SJ, Kim HJ, Lee SJ, Park YJ, Lee J, You HK (2006) Effects of nicotine on proliferation and osteoblast differentiation in human alveolar bone marrow-derived mesenchymal stem cells. Acta Biochim Biophys Sin (Shanghai) 38:874-882

11. Henemyre CL, Scales DK, Hokett SD et al (2003) Nicotine stimulates osteoclast resorption in a porcine marrow cell model. $\mathrm{J}$ Periodontol 74:1440-1446

12. Tanaka H, Tanabe N, Shoji M et al (2006) Nicotine and lipopolysaccharide stimulate the formation of osteoclast-like cells by increasing macrophage colony-stimulating factor and prostaglandin E2 production by osteoblasts. Life Sci 78:1733-1740

13. Jimi E, Shuto T, Koga T (1995) Macrophage colony-stimulating factor and interleukin-1a maintain the survival of osteoclast-like cells. Endocrinology 136:808-811

14. Lee SK, Chung JH, Choi SC et al (2013) Sodium hydrogen sulfide inhibits nicotine and lipopolysaccharide-induced osteoclastic differentiation and reversed osteoblastic differentiation in human periodontal ligament cells. J Cell Biochem 114:1183-1193

15. Theoleyre S, Wittrant Y, Tat SK, Fortun Y, Redini F, Heymann D (2004) The molecular triad OPG/RANK/RANKL:Involvement in the orchestration of pathophysiological bone remodeling. Cytokine Growth Factor Rev 15:457-475

16. Wu LZ, Duan DM, Liu YF, Ge X, Zhou ZF, Wang XJ (2013) Nicotine favors osteoclastogenesis in human periodontal ligament cells co-cultured with CD4(+) T cells by upregulating IL-1 $\beta$. Int J Mol Med 31:938-942

17. Lee HJ, Pi SH, Kim Y et al (2009) Effects of nicotine on antioxidant defense enzymes and RANKL expression in human periodontal ligament cells. J Periodontol 80:1281-1288

18. Sorkhdini P, Moslemi N, Jamshidi S, Jamali R, Amirzargar AA, Fekrazad R (2013) Effect of hydrosoluble chlorine-mediated antimicrobial photodynamic therapy on clinical parameters and cytokine profile in ligature-induced periodontitis in dogs. J Periodontol 84:793-800

19. Oda S, Nitta H, Setoguchi T, Izumi Y, Ishikawa I (2004) Current concepts and advances in manual and power-driven instrumentation. Periodontol 36:45-58

20. Braham P, Herron C, Street C, Darveau R (2009) Antimicrobial photodynamic therapy may promote periodontal healing through multiple mechanisms. J Periodontol 80:1790-1798

21. Garcia VG, Fernandes LA, Macarini VC et al (2011) Treatment of experimental periodontal disease with antimicrobial photodynamic therapy in nicotine-modified rats. J Clin Periodontol 38:1106-1114

22. Garcia VG, Gualberto Júnior EC, Fernandes LA et al (2013) Adjunctive antimicrobial photodynamic treatment of experimentally induced periodontitis in rats with ovariectomy. J Periodontol 84: 556-565

23. Yamano S, Berley JA, Kuo WP et al (2010) Effects of nicotine on gene expression and osseointegration in rats. Clin Oral Implants Res 21:1353-1359 
24. Garcia VG, Longo M, Gualberto Júnior EC et al (2014) Effect of the concentration of phenothiazine photosensitizers in antimicrobial photodynamic therapy on bone loss and the immune inflammatory response of induced periodontitis in rats. J Periodontal Res 49:584-594

25. Ström JO, Theodorsson E, Theodorsson A (2008) Order of magnitude differences between methods for maintaining physiological 17 beta-oestradiol concentrations in ovariectomized rats. Scand J Clin Lab Invest 68:814-822

26. Raval AP, Hirsch N, Dave KR, Yavagal DR, Bramlett H, Saul I (2011) Nicotine and strogen synergistically exacerbate cerebral ischemic injury. Neuroscience 181:216-225

27. Bosco AF, Bonfante S, de Almeida JM, Luize DS, Nagata MJ, Garcia VG (2007) A histologic and histometric assessment of the influence of nicotine on alveolar bone loss in rats. J Periodontol 78: $527-532$

28. Hapidin H, Othman F, Soelaiman IN, Shuid AN, Luke DA, Mohamed N (2007) Negative effects of nicotine on boneresorbing cytokines and bone histomorphometric parameters in male rats. J Bone Miner Metab 25:93-98

29. Müller Campanile VS, Giannopoulou C, Campanile G, Cancela JA, Mombelli A (2015) Single or repeated antimicrobial photodynamic therapy as adjunct to ultrasonic debridement in residual periodontal pockets: clinical, microbiological, and local biological effects. Lasers Med Sci 30:27-34

30. Tardivo JP, Del Giglio A, de Oliveira CS et al (2005) Methylene blue in photodynamic therapy: from basic mechanisms to clinical applications. Photodiagnosis Photododyn Ther 2:175-191

31. Ochsner M (1997) Photophysical and photobiological processes in the photodynamic therapy of tumors. J Photochem Photobiol B 39:1-18
32. Kömerik N, Nakanishi H, MacRobert AJ, Henderson B, Speight P, Wilson M (2003) In vivo killing of Porphyromonas gingivalis by toluidine blue-mediated photosensitization in an animal model. Antimicrob Agents Chemother 47:932-940

33. Wainwright M (1998) Photodynamic antimicrobial chemotherapy (PACT). J Antimicrob Chemother 42:13-28

34. Usacheva M, Teichert MC, Biel MA (2001) Comparison of the methylene blue and toluidine blue photobactericidal efficacy against gram-positive and gram-negative microorganisms. Lasers Surg Med 29:165-173

35. Komerik N, Wilson M, Poole S (2000) The effect of photodynamic action on two virulence factors of gram-negative bacteria. Photochem Photobiol 72:676-680

36. Asagiri M, Takayanagi H (2007) The molecular understanding of osteoclast differentiation. Bone 40:251-264

37. Cochran DL (2008) Inflammation and bone loss in periodontal disease. J Periodontol 79:1569-1576

38. Graves DT, Cochran D (2003) The contribution of interleukin-1 and tumor necrosis factor to periodontal tissue destruction. J Periodontol 74:391-401

39. Lerner UH (2006) Inflammation-induced bone remodeling in periodontal disease and the influence of postmenopausal osteoporosis. J Dent Res 85:596-607

40. Boyle WJ, Simonet WS, Lacey DL (2003) Osteoclast differentiation and activation. Nature 423:337-342

41. Séguier S, Souza SL, Sverzut AC et al (2010) Impact of photodynamic therapy on inflammatory cells during human chronic periodontitis. J Photochem Photobiol B 101:348-354 